\title{
Evolutionary Computation Methods in Image Watermarking
}

\author{
Ahmed.A.Saleh \\ Department of Math and Computer Science \\ Alexandria University \\ EGYPT
}

\author{
M.A.Abdou \\ Associate Professor \\ Informatics Research Institute, City for Scientific \\ Research \& Technology Applications \\ Adjunct Associate Professor, Pharos University in \\ Alexandria, EGYPT
}

\begin{abstract}
Watermarking can be described as a desirable alternative solution for copyright protection of digital materials like images, video and audio. This paper introduced a modified watermarking method in Wavelet domain to get better performance. Particle Swarm Optimization (PSO) is used to find the best DWT coefficients in the HL sub - band for embedding the watermark sequence and PSO fitness function can be formed from the watermarked image with the best possible quality, and proved execution time.
\end{abstract}

\section{Keywords}

Watermarking, discrete wavelet transform (DWT), particle swarm optimization (PSO), and translation map (TM), round map (RM).

\section{INTRODUCTION}

Digital Image watermarking, can be described as a pattern of bits inserted into a digital image, video or audio file that helps to identify the file's copyright information (the author, the rights, etc.) [1]. Digital watermark algorithms should satisfy two important properties. First,the embedded watermark does not affect the quality of the image (i.e. The original image and watermarked image are the same in many tests like Human vision Test) and should be totally invisible. Second, the watermark is robust to common image attacks like lossy compression, linear or non-linear filtering, cropping, noise attacks, etc... Any trails to remove the watermark should affect watermarked image quality $[7,8]$. The watermark may be a visible (printed) or non-visible. The purpose of digital watermarks is to provide copyright protection. Previous techniques could be classified into two classes depending on the need to the presence of the original image or not. Some schemes reported as methods required the original image for detection and others extract the watermark without using the original image [8]. Most common watermarking techniques use an additive watermark, i.e. the watermark is added to the image pixels in the spatial domain or to the coefficient in the transform domain. In this paper, the focusing was on embedding watermark to the coefficient of the discrete wavelet transform. Here in this paper, a wavelet based watermarking method that adds the values of watermark image in horizontal details sub-band of the DWT of the original image [1]. The main advantage of using the DWT is that the human eyes are not sensitive to small changes in edges and textures of an image but are very sensitive to small changes in the smooth parts of an image also embedding watermark in wavelet domain is results more robust image. With the DWT, the edges and textures are usually well connected to the high frequency sub-bands, such as $\mathrm{HH}, \mathrm{LH}$,
HL, etc. Large coefficients in these bands usually indicate edges in an image. Therefore, adding watermarks to these large coefficients is difficult for the human eyes to perceive. The watermarking technique has multi-steps shown in Figure 1. Large number of researchers focus on the watermarking Algorithm used and how to improve it by decreasing time to embed watermark as well as the lack of a difference between the original image and the watermarked image.

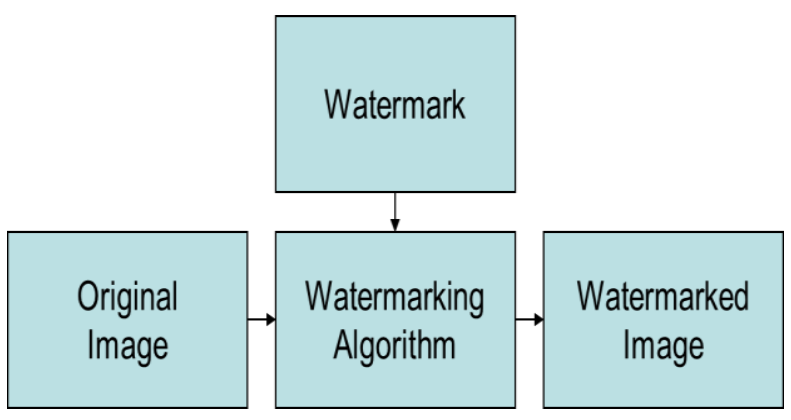

Fig 1. Image watermarking Block Diagram

DWT can be implemented using digital filters and downsamplers. The original image is decomposed into four quadrant bands after decomposition. The four quadrants contain approximation sub-band (LL), horizontal detail subband (LH), vertical detail sub-band (HL) and a diagonal detail sub-band $(\mathrm{HH})$. This process can be repeatedly applied to the approximation sub-band to generate the next scale of wavelet coefficients. The process continues until some final scale is reached [2] .In 2010, Hwei-Jen Lin , Che-Wei Lu and Jih-Pin Yeh [3], presented a robust watermarking method based on Discrete Wavelet transform (DWT), Hwei-Jen Lin [3] shows an improvement in V. Aslantas et al [4] technique that presented a "fragile watermarking method based on the Discrete Cosine transform (DCT) and Genetic Algorithm (GA)". However, the Discrete Cosine Transform has a degree of complexity in the calculation, also the GA process need very long time to get the best solution, and the watermark embedded in the right bit of low frequency coefficients .i.e. DCT and GA need longer time in processing and the watermarked images don't resist certain attacks like cropping and noise attack. Therefore, Hwei-Jen Lin [3] tried to overcome these problems. By using DWT instead of DCT and Particle Swarm Optimization PSO instead of Genetic Algorithm GA to get better translation map. There, watermark is embedded in the LSB (right bit) of the coefficient of the HL sub-band. This work will be differed from Hwei-Jen Lin [3] that the Particle Swarm Optimization (PSO) will be applied in wavelet domain instead of the spatial domain. Several steps are applied to implement this modified algorithm to achieve high image quality, better execution time, and more robust 
against attacks. This paper is organized as follows. In Section2, The PSO and its mechanism will be introduced. In Section 3, the proposed method in detail will be introduced. In section 4, the effect of higher DWT decomposition will be shown. In section 5, the experimental results of work will be shown. Finally, Section 6 gives the conclusion.

\section{PARTICLE SWARM OPTIMIZATION (PSO)}

Particle swarm optimization is a recent stochastic, populationbased evolutionary computation algorithm for problem solving. The particle swarm optimization algorithm was first described in 1995 by James Kennedy and Russell C. Eberhart. The basic idea of PSO came from the research on the behavior of bird swarms looking for food. Every particle always follows the two best positions the best position in the whole swarm and itself in iteration computation [5].

\subsection{Mechanism of PSO}

The particles start at an initial position may be random position and try to search for the maximum or minimum of a fitness function by searching through the search space. Each particle changes its position and velocity in the search space by knowing its velocity and the position where good solutions have already been found by the particle itself or its neighboring particles. When the whole swarm is defined as the neighborhood it is called the global version, and otherwise it is called the local version. Equation 1 and 2 describe how each particle calculates its next position and velocity [6].

Generate initial swarm and velocities randomly

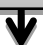

Evaluate the initial swarm, using the initial

performance as the initial personal best and using that to find the initial neighborhood best

Update velocity of particles using personal best and neighborhood best

Apply velocities to positions of particles

Apply velocities to positions of particles

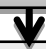

Evaluate new particle positions

If the fitness is not deterministic, also reevaluate the personal best positions

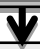

Find the new personal best for each particle and use that to find the new neighborhood bests

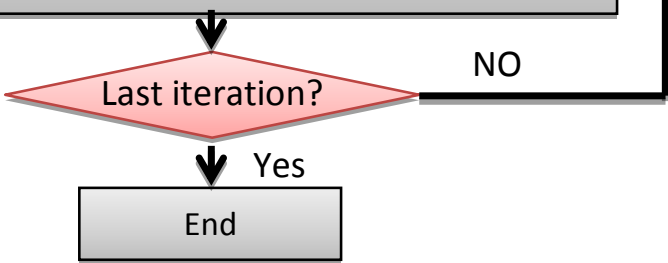

$$
\begin{aligned}
v_{i d}=w \cdot v_{i d}+c_{1} \cdot r_{1} \cdot\left(\text { gbest }-x_{i d}\right) & \\
& +c_{2} \cdot r_{2} \cdot\left(\text { lbest }_{i}-x_{i d}\right) \quad \text { Equation } 1
\end{aligned}
$$

$x_{i d}=x_{i d}+v_{i d}$

Equation 2

Each particle updates its velocity by using Equation $1 . c_{1}$ and $c_{2}$ are cognitive coefficients and $\boldsymbol{r}_{1}$ and $\boldsymbol{r}_{2}$ are random numbers between $[0,1]$. Gbest is the global best position and lbest $t_{i}$ is the individual best of a specific particle $i$.

And Equation 2 updates each particle's position in the search space. And the figure 2 shows the algorithm

\section{THE PROPOSED ALGORITHM}

The watermark was embedded in the detailed horizontal subband of the DWT. The HL sub-band is divided into $4 \times 4$ nonoverlapping blocks, at each block the four coefficients in the inverse diagonal are selected to embed watermark in the LSBs coefficients for each block [3]. After that the HL sub-band is decomposed into separate squared blocks of size $8 \times 8$. In every square block, PSO searches for the translation map that readjusted HL parameters from real to integer numbers. The translation map operates as follows: a value zero in the

translation map, floored decimal fraction of HL mods corresponding value only. For each bit with a value one in the translation map, ceiled a decimal fraction of HL sub-band mods corresponding value only as shown in Equation 3. Inverse DWT (IDWT) is then executed; then the map round is calculated to set real numbers to IDWT image for the corresponding integer number with respect to its round map (binary map). The map is calculated round of pixel values from IDWT image, if the decimal part of the pixel value greater than or equal to 0.5 , then the corresponding value in the bitmap of round 1 . If the decimal part of the pixel value less than 0.5 , then the corresponding value in bit 0 round map as shown in equation 4. Values are calculated for the final image watermarked with equation 5 . Figure 3 . Describes the algorithm.

The steps of the proposed method are listed as:

a) For to the original picture $(F)$, the $1^{\text {st }}$ level Discrete Wavelet transform (DWT) is applied.

b) The watermark image is divided into separated squared blocks each of size two times two.

c) The HL sub-band is also divided into separated squared blocks each of size $4 \times 4$ and begin to embed the four watermark bits to the LSBs of the four coefficients in the inverse diagonal for each HL sub-band block.

HL mods and then divided into separated squared blocks of size 8 times eight.

d) The PSO is used to train and search the optimal translation map for every squared block. $H L(m, n)$

$=\left\{\begin{array}{c}\operatorname{Ceil}(H L(m, n)), \operatorname{MAP}(m, n)=1 \\ \operatorname{Floor}(H L(m, n)), \operatorname{MAP}(m, n)=0\end{array}\right.$

Equation 3

e) For the embedded image, the Inverse Discrete wavelet transform is performed on it to get IDWT image

Fig 2. Describing the evolutionary optimization loop used by PSO. 
f) Calculate the round map (RM) from the following

$R M(i, j)$

$=\left\{\begin{array}{lr}1, \mid I D W T(i, j)-\text { floor }(I D W T(i, j)) \mid \geq 0.5 \\ 0, & \text { otherwise }\end{array}\right.$

Equation 4

g) Then the watermarked image $\boldsymbol{I}_{A D J}$ is calculated using the round map from the following rules

$I_{A D J}(i, j)$

$=\left\{\begin{aligned} \operatorname{Ceil}(\operatorname{IDWT}(i, j)), & \text { roundMap }(i, j)=1 \\ \text { Floor }(I D W T(i, j)), & \text { roundMap }(i, j)=0\end{aligned}\right.$ Equation 5

First create the random swarms of particles which are needed to apply the PSO algorithm to the proposed technique. Each particle is represented as a string, say $C_{i}^{k}=c_{i 1} c_{i 2} \ldots c_{i m}, i=1,2, \ldots, d$ where $\mathrm{d}$ is the swarm size (number of particles). Thus, each particle is placed randomly in m-dimensional space as a candidate solution. Hwei-Jen Lin [5], particle size is the same as that of the translation map (eight times eight); that is, 64. Each element in the string

to produce the new position of each particle. After the PSO, all of the 256 squared blocks of IDWT have its best translation map.

$$
\begin{aligned}
& \text { Fitness function }=64 \\
& -\sum_{i=1}^{8} \sum_{j=1}^{8}\left|I(i, j)-I^{\prime}(I, J)\right| \text { Equation } 6 \\
& \sum_{i=1}^{4}\left|W_{i}-W_{i}^{\prime}\right| \\
& \text { Equation } 7 \\
& U_{i}^{k}=\text { pbest }_{i}+\text { gbest }-2 C_{i}^{k} \\
& \text { Equation } 8 \\
& C_{i}^{k+!}= \begin{cases}0 & \text { if } U_{i}^{k}<0 \\
1 & \text { if } U_{i}^{k}>0 \\
C_{i}^{k} & \text { if } U_{i}^{k}=0\end{cases}
\end{aligned}
$$

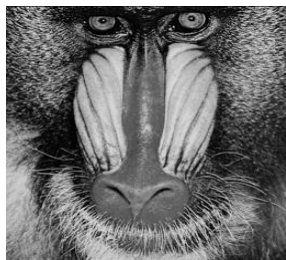

Original Image

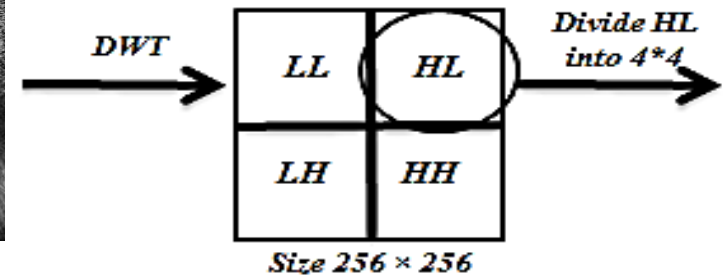

Watermark Image

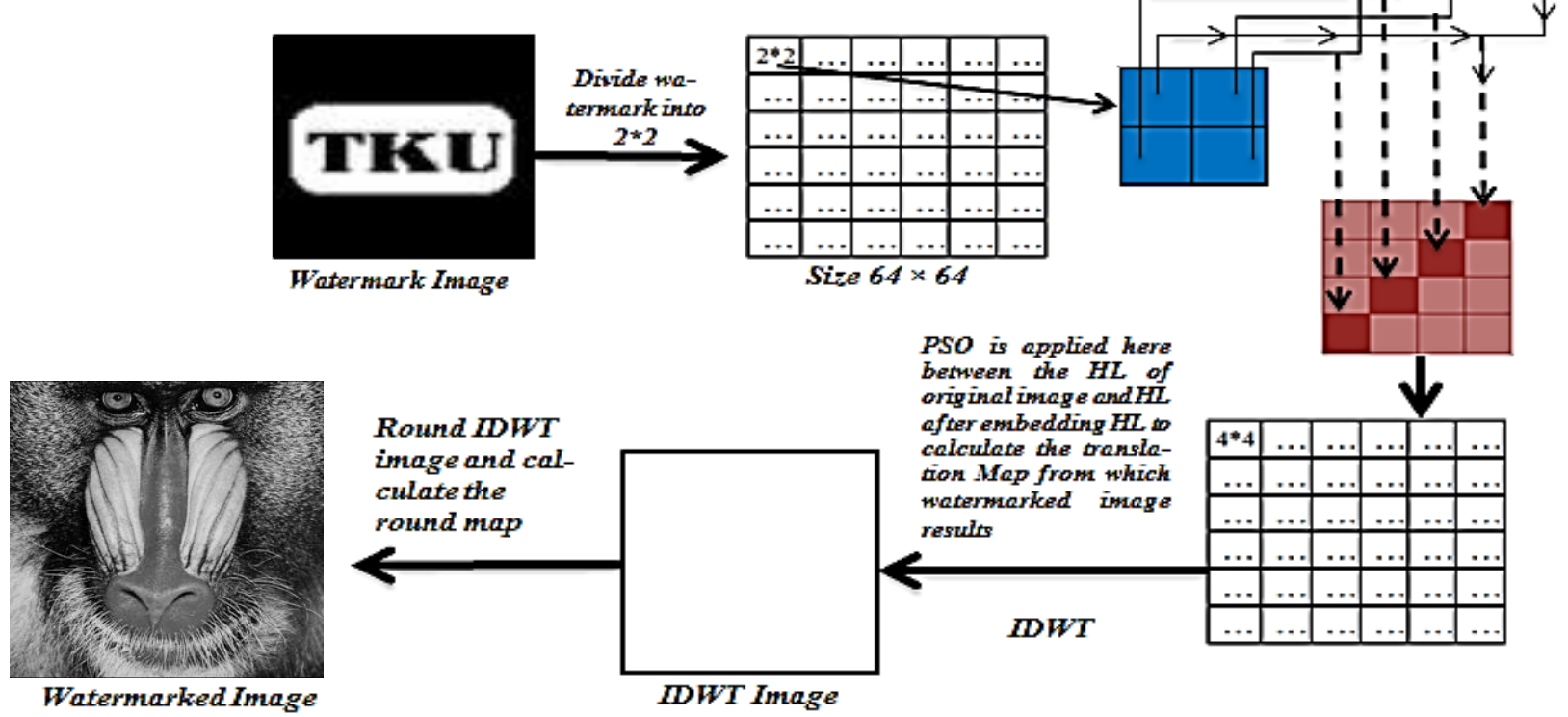

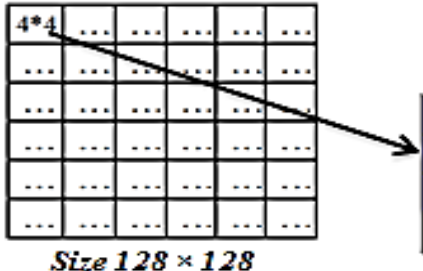

Size $128 \times 128$
Size $4 \times 4$
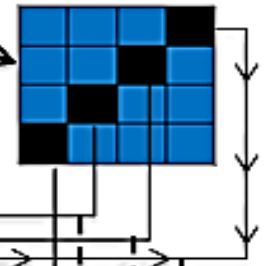

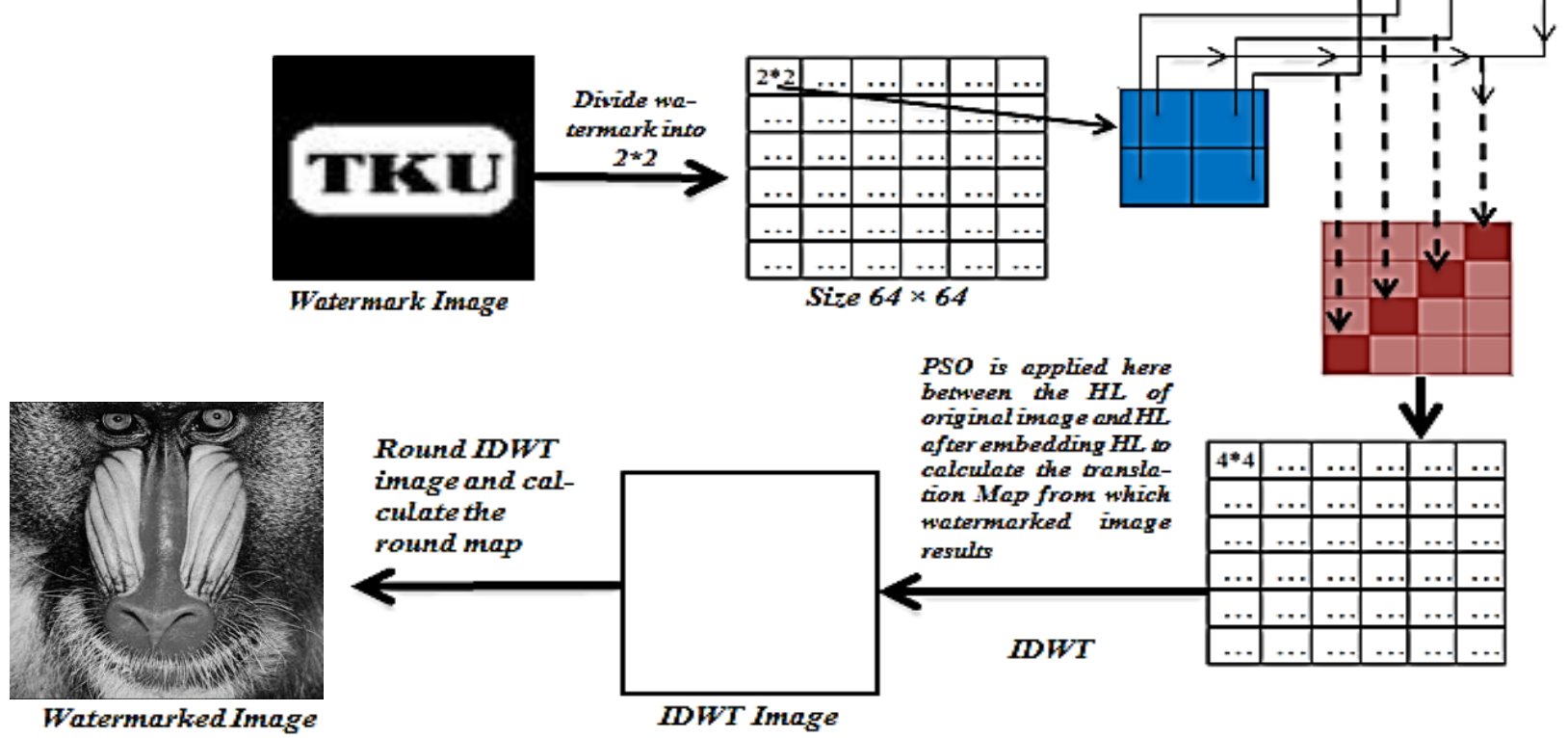

Fig 3. Image watermarking flow graph

represents particle corresponds to a map bit. Population size is $\mathrm{d}$ is eighty, and max number of iterations $=10$. The fitness function is declared fit in equation 6 where $\boldsymbol{I}$ is a squared block of HL sub-band comes from the original image and I' is a squared block of modified HL sub-band included. Under constraints to control PSO as shown in equation 7 where $\mathrm{W}^{\prime}$ is a block of watermark extracted relevant to $\mathrm{W}$ block which corresponds to the original watermark. First, the velocity of each particle is updated as shown in equation 8 , where pbest $t_{i}$ is the particle previous position of each particle and gbest is the best global mode between all particles. The position is updated from the formula described in equation 9 which used

\section{To extract the embedded watermark:}

a) For the watermarked image, the 1st level Discrete Wavelet transform (DWT) is applied.

b) From the HL sub-band coefficients, begin to extract the embedded bits, divide HL sub-band into $4 * 4$ squared blocks, then from the inverse diagonal coefficient of each block, and the watermark can be extracted. 


\section{Effect of higher Wavelet decomposition}

Another new technique where the $2^{\text {nd }}$ wavelet decomposition is applied to the original (Host) image. The steps could be summarized as:

a) Apply $2^{\text {nd }}$-Level Discrete Wavelet Transform (DWT) on an Original image $(\boldsymbol{F})$.

b) The watermark image is divided into separated squared blocks each of size two times two.

c) The HL2 sub-band is also divided into separated squared blocks each of size four times four and begins to embed the four watermark bits to the LSBs of the four coefficients in the inverse diagonal for each HL sub-band block.

d) HL2 mods and then divided into separated squared blocks of size eight times eight.

e) And then, the PSO is used to train and search the optimal translation map for every squared block.

$H L 2(m, n)$

$=\left\{\begin{array}{l}\operatorname{Ceil}(H L 2(m, n)), \operatorname{MAP}(m, n)=1 \\ \operatorname{Floor}(H L 2(m, n)), \operatorname{MAP}(m, n)=0\end{array}\right.$

Equation 10

f) Perform Inverse Discrete Wavelet Transform (DWT) on the embedded image to get an image (IDWT image).

g) Calculate the round map from the following

$R M(i, j)$

$=\left\{\begin{array}{l}1, \mid I D W T(i, j)-\text { floor }(\operatorname{IDWT}(i, j)) \mid \geq 0.5 \\ 0, \quad \text { otherwise }\end{array}\right.$ Equation 11

h) Then the watermarked image $\boldsymbol{I}_{\boldsymbol{A D J}}$ is calculated using the round map from the following rules

$I_{A D J}(i, j)$

$=\left\{\begin{aligned} \operatorname{Ceil}(\operatorname{IDWT}(i, j)), & \operatorname{roundMap}(i, j)=1 \\ \text { Floor }(\operatorname{IDWT}(i, j)), & \operatorname{roundMap}(i, j)=0\end{aligned}\right.$ Equation 12

\section{To extract the embedded watermark:}

a) For the watermarked image, the $2^{\text {nd }}$ level Discrete Wavelet transform (DWT) is applied.

b) From the HL2 sub-band coefficients begin to extract the embedded bits, divide HL sub-band into $4 * 4$ squared blocks, then from the inverse diagonal coefficient of each block, and the watermark can be extracted.

This developed algorithm (increasing wavelet decompositions) gives better time, but very bad in watermark extraction comparing with Hwei-Jen Lin's algorithm.

\section{OBTAINED RESULTS}

Now, this work will move in two parallel ways. The proposed PSO method applied in wavelet domain is compared with [3], which applies optimization in time domain. This is usually a debate whether Wavelet Transformation (WT) domain is a better solution to various image processing problems solving. The algorithm that has been implemented was presented in [3] to compare and evaluate the performance of this proposed method. The comparison will attack execution time, PSNR, MSE and NC calculated by substitution in Equation 13,

Equation 14 and Equation 15 between original and watermarked images in both algorithms. Also calculated between Original watermark and Extracted watermark.

The practice test is carried out on PC has the following specification: Operating System: Windows 7 Ultimate 32-bit, Processor: Intel (R) Core (TM) 2 Duo CPU T6570 @ 2.10GHz (2 CPUs), Memory: 2048MB RAM and MATLAB version: 7.14.0.739 (R2012a). In the experiments, in the practical work, a large number of gray scale images have been represented as host images and many binary images have been represented as the watermark image. The host images are of size $256 \times 256$ while the watermark images are of size $64 \times$ 64. Two host images and one watermark are selected to show the results on them as shown in Figure 4. Some statistical measurements like Image Quality Measures like Mean Square Error (MSE), Peak Signal to Noise Ratio (PSNR) and Normalized Correlation (NC) will be used to compare performance. Formulas for MSE, PSNR and NC are shown in equations 13,14 and 15 .The value greater than $30 \mathrm{~dB}$ for PSNR is acceptable. The watermarked images shown in figure 5 and figure 6 .

$$
\begin{aligned}
& M S E=\frac{1}{W \times H} \sum_{i=1}^{W} \sum_{j=1}^{H}\left(F(i, j)-F^{\prime}(i, j)\right)^{2} \\
& P S N R=10 \times \log _{10}\left(\frac{255^{2}}{M S E}\right) \\
& N C=\sum_{i=1}^{H_{0}} \sum_{j=1}^{W_{0}} \frac{W(i, j) \times W^{\prime}(i, j)}{\left(H_{0} \times W_{0}\right)}
\end{aligned}
$$

Equation 13

Equation 14

Equation 15

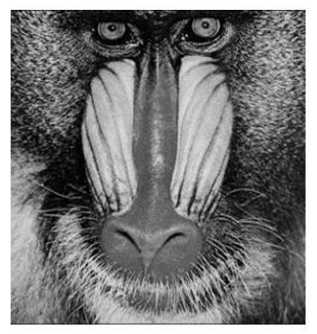

Image (a)

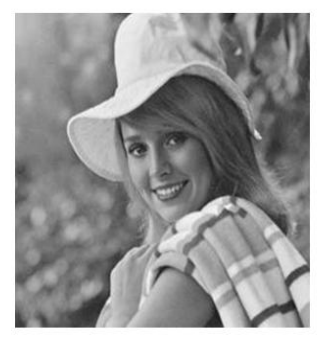

Image (b)

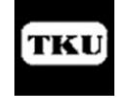

Fig 4. Two host images and one watermark

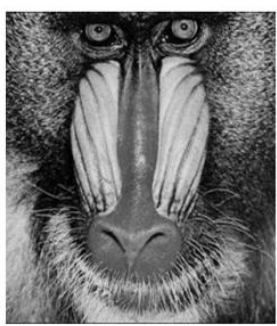

watermarked (a)

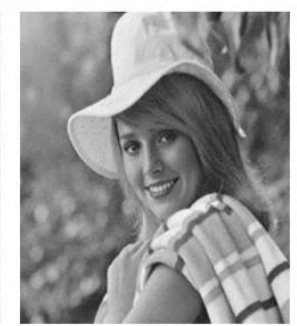

watermarked (b)
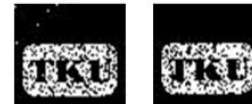

Fig 5. Developed algorithm resulted image from watermarking \& Extracted watermark 


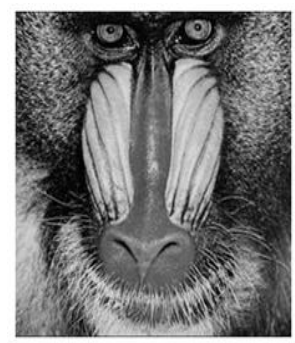

watermarked (a)

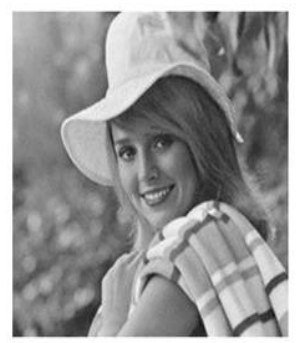

watermarked (b)

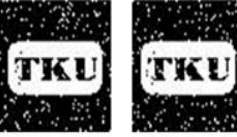

Extracted watermark from image a \& image b respectivly
Fig 6. Hwei-Jen Lin's Method resulted image from watermarking \& Extracted watermark

Table 1. PSNR, the run time, NC without images attacks for the this method and Compared method

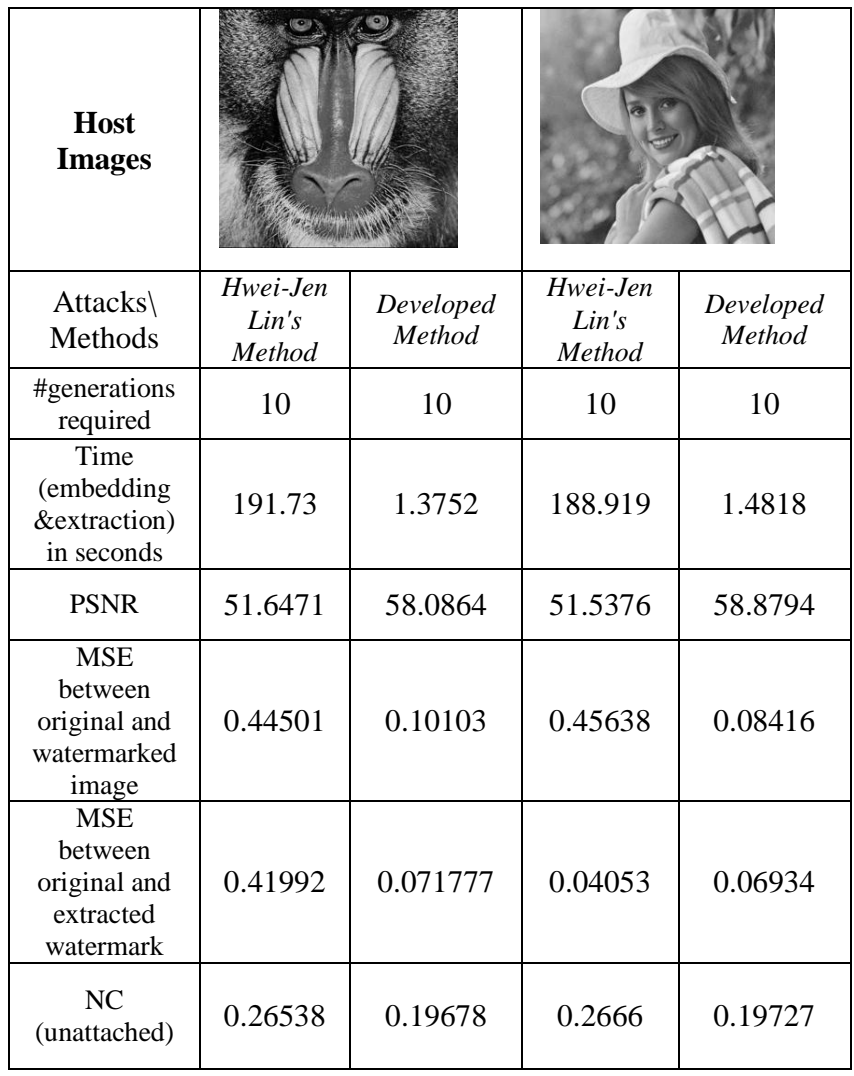

Table 1, compares the time of watermarking between HweiJen Lin's and developed Method, also PSNR between original (host) image and watermarked (stego) image, Moreover MSE values between watermarked images and the original image and MSE between the original watermark and extracted watermark. NC values of the extracted watermarks. For this paper method and Hwei-Jen Lin's method, The Comparison shows that this developed an algorithm reduces time by $\mathbf{1 4 0} \%$ to embed the watermark with respect to and Hwei-Jen Lin's. From the image quality point of view, the table shows that the proposed method gives good results in both visual and analytical tests. The MSE has been reduced by $20 \%$ and the PSNR has been increased to $10 \%$.

Table 2, shows that the image noise attack of this paper watermarked image and Hwei-Jen Lin's watermarked image At noise density 0.01 , the MSE has increased by $13 \%$; however the extracted watermark image is satisfactory from visualization tests. At noise density 0.05 , the MSE has increased by $11 \%$; however the extracted watermark image is satisfactory from visualization tests.

Table 2. MSE of extracted watermark from an image which is attacked by salt $\&$ pepper noise for this method and

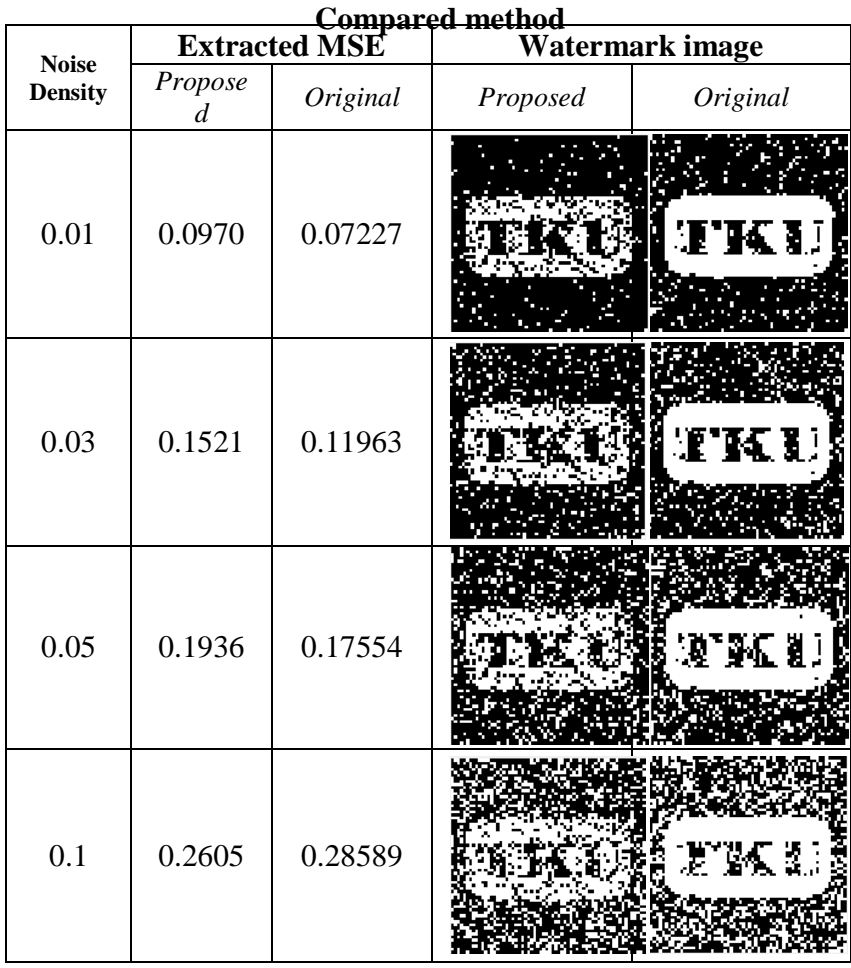

\section{Conclusion}

In this paper, a developed technique based on Hwei-Jen Lin's Method has been introduced. A different scenario applied in wavelet domain that embeds the watermark in WT coefficients is tested. An optimal translation map has been reached using PSO in extremely efficient execution time when compared with [3]. WT domain watermarking that replaces spatial domain method has reduced time in watermark embedding and extraction by $\mathbf{1 4 0} \%$. Furthermore, PSNR has been improved by $\mathbf{1 0} \%$, which means that better image quality has been obtained. 


\section{REFERENCES}

[1] Xia, X.G., C. Boncelet, and G. Arce, Wavelet transform based watermark for digital images. THE INTERNATIONAL ONLINE JORNAL OF OPTICS, 1998. 3(12).

[2] Mahmoud, K., S. Datta, and J. Flint, Frequency Domain Watermarking: An Overview. The International Arab Journal of Information Technology, 2005. 2(1): p. 33-47.

[3] Lin, H.-J., C.-W. Lu, and J.-P. Yeh, DWT-BASED WATERMARKING TECHNIQUE ASSOCIATED WITH TRANSLATION MAPS. International Conference on Business and Information (BAI2010), 2010.

[4] Aslantas, V., S. Ozer, and S. Ozturk, Improving the performance of DCT-based fragile watermarking using intelligent optimization algorithms. Optics Communications, 2009. 282(14): p. 2806-2817.
[5] Kennedy, J. and R. Eberhart. Particle swarm optimization. in Neural Networks, 1995. Proceedings., IEEE International Conference on. 1995.

[6] Rohani, M. and A.N. Avanaki, A Watermarking Method Based on Optimizing SSIM Index by using PSO in DCT Domain 14th International CSI conference (CSICC2009), July 1-2, 2009, Tehran, Iran 2009.

[7] Inoue, H. , Miyazaki, A. and Katsura, T. An image watermarking method based on the wavelet transform ICIP International Conference on 1999.

[8] Rakesh D., Krishna R. and Narendra A. A new waveletbased scheme for watermarking images. ICIP International Conference in 1998 